\title{
Modulation of Human Motor Cortex Excitability by Single Doses of Amantadine
}

\author{
Janine Reis', Daniel John', Antje Heimeroth', Hans-Helge Mueller², Wolfgang H Oertel', Torsten Arndt ${ }^{3}$ \\ and Felix Rosenow*, I \\ 'Department of Neurology, Interdisciplinary Epilepsy Center, Philipps-University, Marburg, Germany; ${ }^{2}$ Institute of Medical Biometry and \\ Epidemiology, Philipps-University, Marburg, Germany; ${ }^{3}$ Bioscientia GmbH Ingelheim, Ingelheim, Germany
}

\begin{abstract}
Amantadine-sulfate has been used for several decades to treat acute influenza A, Parkinson's disease (PD), and acute or chronic druginduced dyskinesia. Several mechanisms of actions detected in vivo/in vitro including N-methyl-D-aspartate (NMDA)-receptor antagonism, blockage of potassium channels, dopamine receptor agonism, enhancement of noradrenergic release, and anticholinergic effects have been described. We used transcranial magnetic stimulation (TMS) to evaluate the effect of single doses of amantadine on human motor cortex excitability in normal subjects. Using a double-blind, placebo-controlled, crossover study design, motor thresholds, recruitment curves, cortical stimulation-induced silent period (CSP), short intracortical inhibition (ICl), intracortical facilitation (ICF), and late inhibition $(\mathrm{L}-\mathrm{ICl})$ in 14 healthy subjects were investigated after oral doses of 50 and $100 \mathrm{mg}$ amantadine with single and paired pulse TMS paradigms. Spinal cord excitability was investigated by distal latencies and M-amplitudes of the abductor digiti minimi muscle. After intake of amantadine, a significant dose-dependent decrease of ICF was noticed as well as a significant increase of L-ICl as compared to placebo. The effect on ICF and L-ICl significantly correlated with amantadine serum levels. ICI was slightly increased after amantadine intake, but the effect failed to be significant. Furthermore, amantadine had no significant effects on motor thresholds, MEP recruitment curves, CSP, or peripheral excitability. In conclusion, a low dose of amantadine is sufficient in modulating human motor cortex excitability. The decrease of ICF and increase of L-ICl may reflect glutamatergic modulation or a polysynaptic interaction of glutamatergic and GABA-ergic circuits. Although amantadine has several mechanisms of action, the NMDA-receptor antagonism seems to be the most relevant effect on cortical excitability. As $\mathrm{L}-\mathrm{ICl}$ can be influenced by this type of drug, it may be an interesting parameter for studies of motor learning and use-dependent plasticity.

Neuropsychopharmacology (2006) 3 I, 2758-2766. doi: I 0. I 038/sj.npp. I 30 I I22; published online I4 June 2006
\end{abstract}

Keywords: amantadine; transcranial magnetic stimulation; Parkinson's disease; motor cortex excitability; glutamate; GABA

\section{INTRODUCTION}

The tricyclic amine amantadine (1-amino-adamantane, AMA) has been widely used since the 1960s for the prophylaxis and treatment of influenza A (Togo et al, 1968). Furthermore, AMA is beneficial for the treatment of Parkinson's disease (PD) (Parkes, 1974), drug-induced parkinsonism, neuroleptic-induced dyskinesia (Kornhuber et al, 1993b), L-Dopa-induced dyskinesia (Verhagen et al, 1998), neuroleptic malignant syndrome, and akinetic hyperthermic Parkinsonian crisis (Kornhuber et al,

\footnotetext{
* Correspondence: Professor Dr F Rosenow, Department of Neurology, Interdisciplinary Epilepsy Center, Philipps-University Marburg, Rudolf-Bultmann-Str. 8, Marburg 35033, Germany, Tel: +49642I 28 65348, Fax: + 49642128 65228,

E-mail: rosenow@staff.uni-marburg.de

Received 31 October 2005; revised 20 April 2006; accepted 8 May 2006

Online publication: 12 May 2006 at http://www.acnp.org/citations/ Npp05 I206050650/default.pdf
}

1993b). The exact pathophysiological correlates for its therapeutic effects in disturbances of the basal ganglia/ motor cortex circuit in humans owing to different kinds of transmitter dysbalance (dyskinesia vs akinetic crisis) have not been investigated yet. Its broad therapeutic applicability alludes to a modulatory, multidirectional action.

Several pharmacological mechanisms of action of AMA have been identified in vitro and in vivo in animals that are thought to contribute to its broad spectrum of mechanisms of action. These include:

1. A low-affine noncompetitive antagonism of the $N$ methyl-D-aspartate (NMDA)-glutamate receptor subtype at the PCP (phencyclidine) binding site, which is localized inside the cation channel (Kornhuber et al, 1991; Parsons et al, 1996) and at the sigma 1-binding site located outside the channel (Kornhuber et al, 1993a). The antagonism of NMDA-induced neuronal currents is use- and voltage-dependent leading to a stabilization of 
channel closed states (Blanpied et al, 2005). Among others this leads to a reduction of acetylcholine (ACh) release from striatal interneurons in vitro (Jackisch et al, 1992; Stoof et al, 1992) by blockage of nicotinergic AChreceptors (Matsubayashi et al, 1997).

2. A direct inhibition of the presynaptic dopamine (DA)reuptake in vitro (Heikkila and Cohen, 1972) and in vivo (Mizoguchi et al, 1994), which results in an increased DA activity. An indirect mechanism of action is the release of DA from striatal dopaminergic neuronal terminals in vitro (Heikkila and Cohen, 1972; Jackisch et al, 1992) and in vivo (Takahashi et al, 1996).

3. An increase of noradrenergic transmission, especially by enhancing the noradrenaline release (Farnebo et al, 1971).

4. A blockage of membrane-bound potassium channels leading to inhibition of the repolarization and therefore an increased membrane excitability (Northover, 1994).

5. A replication inhibition of influenza A virus by blockage of M2 protein ion channel leading to disturbed virus encoating and disturbed $\mathrm{pH}$ regulation (Takeda et al, 2002; Lin et al, 1997).

Amantadine (PK-Merz ${ }^{\circledR}$, Merz Pharmaceuticals GmbH, Frankfurt, Germany) has a complete bioavailability, is rapidly absorbed and maximum plasma levels are reached within 1-12 h after oral administration (Aoki et al, 1979; Aoki and Sitar, 1985). Mean half time is $15 \mathrm{~h}$. The peak plasma concentration after $100 \mathrm{mg}$ AMA sulfate is $0.15 \mu \mathrm{g} /$ ml. AMA elimination is predominantly renal as unchanged compound (90\%). The blood-brain barrier passage of the highly lipophilic drug depends on the degree of saturation. This is supported by toxicological studies, which indicate a main effect in the central nervous system (CNS) (Vernier et al, 1969). The brain (tissue)/serum relation between AMA concentrations is 0.6 (Kornhuber et al, 1995).

With transcranial magnetic stimulation (TMS) main mechanisms of cortical excitability, cortical representation of different muscles (Pascual-Leone et al, 1994), and the pathophysiology of neurological diseases, for example, epilepsy (Hamer et al, 2005), dystonia (Siebner et al, 1998) or PD (Priori et al, 1994), have been previously investigated. Furthermore, the influence of CNS-related drugs with a well-known mode of action was evaluated and differentiated from pathophysiological changes of the disease itself (Liepert et al, 1997; Ziemann et al, 1996a). Additionally, TMS has been used as a tool to characterize the in vivo effects of newly developed drugs on cortical excitability (Reis et al, 2002, 2004; Werhahn et al, 1999). For this purpose, different electrophysiological parameters evoked by single and paired pulse TMS are available to investigate changes in motor cortex excitability after drug intake. Using the paired pulse paradigm, indirect information about subpopulations of intracortical inhibitory and excitatory interneurons can be obtained.

As AMA is a drug which is frequently used in clinical practice and effective in different types of movement disorders (ranging from akinesia to dys-/hyperkinesia), we wanted to study to which extent the motor cortex excitability (either as a direct target for pharmacological influence or as a remote structure in the basal gangliathalamo-cortical loop) is modulated by single doses of
AMA. We aimed to characterize the significance of the proposed different in vitro and in vivo mechanisms of action using TMS. Therefore, we decided to use low therapeutic doses of AMA to enhance the probability to observe drug-specific effects, avoiding unselective effects on transmitter systems of the human cortex revealed at the high-dose range. We hypothesized that AMA would lead to a significant change of cortical excitability as compared to placebo. Based on results of TMS studies of other adamantanes such as memantine (Schwenkreis et al, 1999), it was assumed, that the main effect of AMA on M1 excitability would include antiglutamatergic properties. Thus, we expected changes in paired pulse TMS parameters (intracortical facilitation (ICF) and inhibition (ICI)).

The antiviral properties of AMA (replication blockade of influenza A virus) were not investigated in this study.

\section{SUBJECTS AND METHODS}

We used single and paired-pulse TMS to investigate which of the mechanisms of action of AMA detected in vitro and in vivo are relevant for the modulation of human motor cortex excitability and, therefore, presumably for the main action on the CNS.

\section{Subjects}

Fourteen healthy volunteers (six women and eight men, mean age $25 \pm 2.8$ years, range $21-33$ years) participated in a randomized double-blind crossover study. All subjects were right-handed, as rated by the Edinburgh Handedness Inventory (Oldfield, 1971). None of the participants had a medical, neurological, or psychiatric illness as assessed by a general and neurological examination, a self-designed 22 items questionnaire screening the medical and psychiatric history as well as contraindications for TMS or amantadine treatment, a routine electrocardiogram, a Mini Mental Status Examination, and a grooved pegboard test (Lafayette Instruments $\left.{ }^{\circledR}\right)$. The subjects were asked not to take any neuro- or psychoactive drugs including alcohol starting 4 days before the first session and for the period of the measurements (maximum 28 days). The study was performed according to the Declaration of Helsinki and approved by the ethics committee of the Philipps-University Marburg. All volunteers gave their written informed consent.

\section{Methods}

Experimental procedures were based on those described previously (Kujirai et al, 1993; Reis et al, 2004).

Motor-evoked potentials (MEPs) were recorded with surface EMG from the right abductor digiti minimi muscle $(\mathrm{ADM})$ in a belly-tendon-montage, contralateral to the left motor cortex. The raw signal was amplified, band passfiltered $(20 \mathrm{~Hz}$ to $10 \mathrm{kHz}$ ), digitized (analog/digital rate $40 \mathrm{kHz}$ ), and recorded onto a PC using a data collection and averaging program (Magnetix ${ }^{\circledR}$, Center for Sensorimotor Research, Munich, Germany) for offline analysis.

Both rest and active conditions were studied in all subjects, monitored by visual (EMG) and auditory feedback. During the active condition, subjects contracted the ADM 
voluntarily with approximately $20 \%$ of maximum force. Between the trials, subjects relaxed for $5 \mathrm{~min}$ to avoid fatigue.

TMS was delivered through a focal figure-of-eight shaped magnetic coil ( $90 \mathrm{~mm}$ external loop diameter) connected to two Magstim 200 magnetic stimulators via a BiStim-module (all Magstim, Whitland, Dyfed, UK). Subjects were seated in an armchair with the head fixed in a plastic foam headrest. The coil was placed flat on the skull over the left motor cortex at the site optimal for contralateral ADM activation. The current induced in the brain beneath the junction of the coil flowed from posterior to anterior, approximately perpendicular to the assumed line of the central sulcus. This is thought to be the most effective way to activate the corticospinal system transsynaptically (Brasil-Neto et al, 1992). This coil position was marked directly on the scalp to ensure accurate repositioning. In all paired pulse TMS procedures, the interval between trials was randomly changed between 4 and $6 \mathrm{~s}$, in single pulse procedures the inter-trial interval was randomly changed between 8 and $10 \mathrm{~s}$.

\section{Measures of Motor Cortex Excitability}

Several TMS measures were used to investigate motor cortex excitability:

1. The amplitudes of the MEPs were measured peak-topeak. The resting motor threshold (RMT) was defined as the lowest stimulator output intensity needed to induce an MEP greater than $50 \mu \mathrm{V}$ peak-to-peak amplitude in at least five of 10 consecutive trials, using a step-by-step intensity resolution of $1 \%$ of the maximal stimulator output. The active motor threshold (AMT) was defined as the lowest stimulator output intensity needed to induce an MEP of more than $50 \mu \mathrm{V}$ in the moderately active ADM muscle (approximately $20 \%$ of maximum force) in at least five of 10 consecutive trials.

2. The MEP recruitment curve depends on the number of activated corticospinal neurons at defined stimulus intensity. By supramaximal electrical stimulation of the ulnar nerve at the wrist, the amplitude of the maximum M-wave $\left(M_{\max }\right)$ of the resting ADM was determined. The received $M_{\max }$ amplitudes obtained by three stimuli were averaged for each subject and set to $100 \%$. Ten TMS stimuli each at intensities of $110,120,130$, and $140 \%$ RMT were applied after that and the averaged MEP amplitude was expressed in \% of $M_{\max }$ to obtain recruitment curves (Reis et al, 2004).

3. The cortical stimulation-induced silent period (CSP) was measured in 20 trials at a stimulus intensity of $130 \%$ AMT in the moderate contracted ADM muscle. CSP duration was defined as the time from TMS stimulus artifact to the first reoccurrence of voluntary EMG activity exceeding prestimulus muscle activity.

4. Short ICI was investigated at interstimulus intervals (ISIs) of 2 and $3 \mathrm{~ms}$, ICF was investigated at ISI 10 and $15 \mathrm{~ms}$. A subthreshold conditioning stimulus applied 1$6 \mathrm{~ms}$ before a test stimulus decreases the resulting MEP amplitude whereas a 7-30 ms ISI produce an increase of the MEP amplitude (Kujirai et al, 1993; Nakamura et al, 1997; Reis et al, 2004). The conditioning stimulus was set to an intensity of $75 \%$ of RMT to exclude changes of excitability in the spinal cord (DiLazzaro et al, 1998). The intensity of the test stimulus was adjusted to produce MEPs of approximately $1.5 \mathrm{mV}$ peak-to-peak amplitude at rest. Late inhibition (L-ICI) was tested at ISI $150 \mathrm{~ms}$ and two equal stimuli of $150 \%$ RMT were delivered. To avoid collecting startle and reflex responses, we excluded the first MEP for each trial from the analysis.

Fifteen trials of the control single test stimuli and 15 paired stimuli of each ISI were recorded, delivered 4-8 s apart in random order generated by the software. The conditioned response was defined as the mean amplitude of the conditioned MEP belonging to the ISI, expressed as percentage of the mean amplitude of the unconditioned test MEP.

5. Peripheral and spinal cord excitability were measured by $\mathrm{M}$ amplitude and $\mathrm{M}$ latency after supramaximal electrical stimulation of the ulnar nerve at the wrist. The results of 15 trials were averaged.

\section{Sessions}

Each subject participated in three sessions. Before the first session, each subject underwent a routine electrocardiogram to exclude cardiac risks for taking AMA, which is known to have rare cardiac side effects.

Stimulation sessions were applied in a pseudo-randomized order, lasted approximately $4 \mathrm{~h}$, and were separated by at least 5 days in order to guarantee return to baseline levels. In order to avoid influence of the subject's biological rhythm on the measurement, all sessions were performed in the afternoon.

Subjects and the investigators performing the measurements and analyzing the raw data were blinded for the order of application. All parameters of motor cortex excitability were measured before (baseline) and $2.5 \mathrm{~h}$ after a single oral dose of either placebo (incl. $10 \mathrm{mg}$ Calcium Sandoz ${ }^{\mathbb{R}}$ Brause, Novartis Consumer Health $\mathrm{GmbH}$, Germany for taste equivalence), 50 or $100 \mathrm{mg}$ of AMA (PK-Merz ${ }^{\mathbb{R}}$ Brause-Tabletten). Doses consisted of white sherbed powder of indistinguishable lemon taste, which was dissolved in water.

The intensity of those side effects associated with the long-term use of AMA was recorded in a semiquantitative fashion $(0=$ not noticed to $3=$ strong) using a standardized questionnaire. A cumulative side effect score was calculated (maximum 54 points). To control for changes in fine and gross motor dexterity and visual-motor coordination, the Grooved Pegboard test (Lafayette Instruments ${ }^{\circledR}$ ) was performed with the dominant and nondominant hand after drug intake and the results were compared to baseline values obtained before drug intake.

$2.5 \mathrm{~h}$ after oral drug ingestion, $10 \mathrm{ml}$ blood serum was taken to evaluate AMA serum levels. Serum was centrifuged $(1750 \mathrm{~g})$ for $10 \mathrm{~min}$ and frozen at $-85^{\circ} \mathrm{C}$ until analysis by an external laboratory (Arndt et al, 2005) (Bioscientia $\mathrm{GmbH}$ Ingelheim, Germany).

\section{Statistical Analysis}

Data are expressed as means \pm SEM. The different measures of motor cortex excitability (MT, MEP recruitment, ICI, 
ICF, and CSP) were analyzed separately. The effect of AMA and placebo on motor cortex excitability measures was compared in an analysis of variance model of repeated measures, with time (pre- and post-intake) and dose (placebo, $50 \mathrm{mg}$ AMA, $100 \mathrm{mg}$ AMA) as the within-subject factor. The three baseline measures were used individually as reference for values obtained at $2.5 \mathrm{~h}$ after drug intake. In general, significance was assumed at $p<0.05$. For post-hoc comparisons (placebo vs $100 \mathrm{mg}$ AMA, baseline $v s$ MED) the Wilcoxon signed-rank test was used and the level of significance was corrected for multiple comparisons using the method of the Bonferroni-Holm.

The AMA serum level/excitability relation of all subjects was evaluated by a two-sided Kendall-Tau-b correlation with the level of significance set to $p<0.05$. For this comparison, only the significant TMS parameters of M1 excitability (ISI 15, ISI 150) were taken into account. To further investigate whether the changes of ISI 15 and ISI 150 were independently targeted by AMA or not, we also used a two-sided Kendall-Tau-b correlation.

\section{RESULTS}

\section{Paired Pulse TMS}

ICF was significantly decreased in a dose-dependent fashion after a single oral dose of 50 and $100 \mathrm{mg}$ AMA as compared to placebo (absolute change ( $\%$ conditioned MEP med-baseline): ISI 15 placebo: 7.77; $50 \mathrm{mg}$ AMA: -12.75 , and 100 mg AMA: -39.83) (Figure 1, Table 1)

At longer ISIs of $150 \mathrm{~ms}$, late inhibition was significantly and dose-dependently increased $2.5 \mathrm{~h}$ after a single oral dose of 50 and $100 \mathrm{mg}$ AMA as compared to placebo (absolute change (\% conditioned MEP med-baseline): ISI 150 placebo: 4.97 ; $50 \mathrm{mg}$ AMA: -5.92 , and $100 \mathrm{mg}$ AMA: -15.69) (Figure 1, Table 1). Furthermore, a significant correlation between the observed changes of ISI 15 and ISI $150(R=0.579, p<0.0001$, Figure 2$)$ was detected.

At short ISIs of 2 and $3 \mathrm{~ms}$, ICI was not significantly altered after a single oral dose of 50 and $100 \mathrm{mg}$ of AMA as compared to placebo (absolute change (\% conditioned MEP med-baseline): ISI 2 placebo: $-1.1,50 \mathrm{mg}$ AMA: -5.28 , and 100 mg AMA: - 14.4; ISI 3 placebo: 3.72, 50 mg AMA: -6.06, and $100 \mathrm{mg}$ AMA: -13.09). However, there was a strong trend toward increased ICI, which seemed to be dosedependent (Figure 1, Table 1).

\section{Motor Thresholds, Recruitment Curves, and CSP}

The motor thresholds (RMT, AMT) were not influenced by a single dose of 50 and $100 \mathrm{mg}$ of AMA as compared to placebo. The MEP amplitudes of the recruitment curves showed a slight increase after 50 and $100 \mathrm{mg}$ of AMA as compared to placebo at 130 and $140 \%$ RMT, these results were not statistically significant.

The duration of the CSP at a stimulation intensity of $130 \%$ AMT remained unchanged after 50 and $100 \mathrm{mg}$ of AMA and compared to placebo (absolute change (ms): CSP 130 placebo: $-4.5,50 \mathrm{mg}$ AMA: 11.0 , and $100 \mathrm{mg}$ AMA: 0.4 ) (Figure 3).
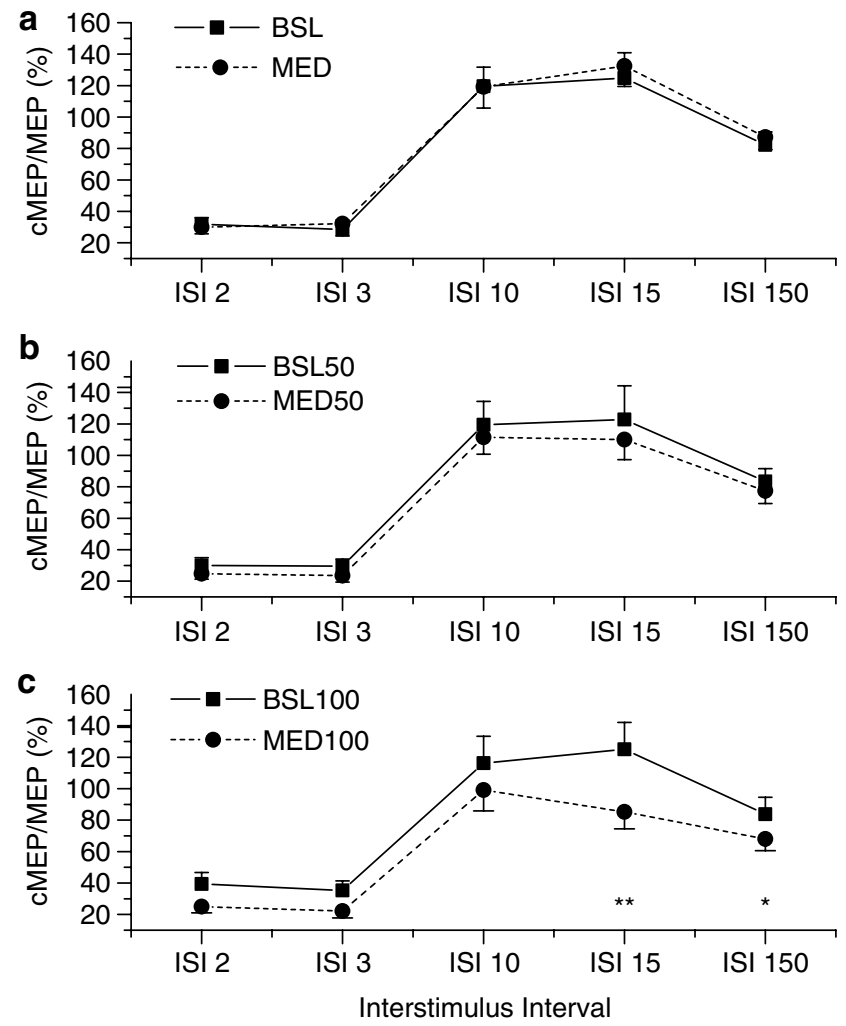

Figure I Average responses after paired TMS (\% of unconditioned responses) of 14 subjects at five different ISIs. Values are expressed as mean \pm SEM. (a) Placebo (MED), (b) $50 \mathrm{mg}$ amantadine (MED 50), (c) $100 \mathrm{mg}$ amantadine (MED 100). $\mathbf{\square}=$ baseline values (BSL), $\mathbf{O}=$ values $2.5 \mathrm{~h}$ after drug intake. ISI I 5 and ISI I 50 were influenced significantly and in a dose-dependent manner after amantadine intake (ANOVA, Wilcoxon's signed-rank test with Bonferroni-Holm adjustment, level of significance: $*=p<0.05$, *** $=p<0.005$ ).

\section{Peripheral Excitability}

The averaged $M$ amplitude and $M$ latency after supramaximal electrical stimulation of the ulnar nerve at the wrist did not differ significantly from baseline values after drug intake in all three sessions.

\section{Amantadine Serum Concentration}

Blood serum was taken $2.5 \mathrm{~h}$ after drug intake. We found a dose-dependent, almost linear increase in AMA serum levels. AMA serum levels after placebo were below $10 \mu \mathrm{g} / \mathrm{l}$ (detection limit). After $50 \mathrm{mg}$ of AMA, the mean serum level was $125.4 \pm 35.1 \mu \mathrm{g} / \mathrm{l}$ (range $87-198 \mu \mathrm{g} / \mathrm{l}$ ) and after $100 \mathrm{mg}$ of AMA the mean serum level was $241.3 \pm 69.4 \mu \mathrm{g} / \mathrm{l}$ (range $130-383 \mu \mathrm{g} / \mathrm{l})$. Taking a therapeutic range of $200-600 \mu \mathrm{g} / \mathrm{l}$ into account, serum levels after the $50 \mathrm{mg}$ dose were below the therapeutic range, whereas $100 \mathrm{mg}$ AMA led to therapeutic serum levels in most of the cases (nine of 14 subjects).

\section{Amantadine Serum Level/Excitability Correlation}

All 42 serum samples were included in the analysis. A significant correlation of AMA serum level and changes of 
Table I Means \pm SEM of the TMS Parameters before (BSL) and after (MED) Intake of Placebo, 50 mg Amantadine, and I00 mg Amantadine

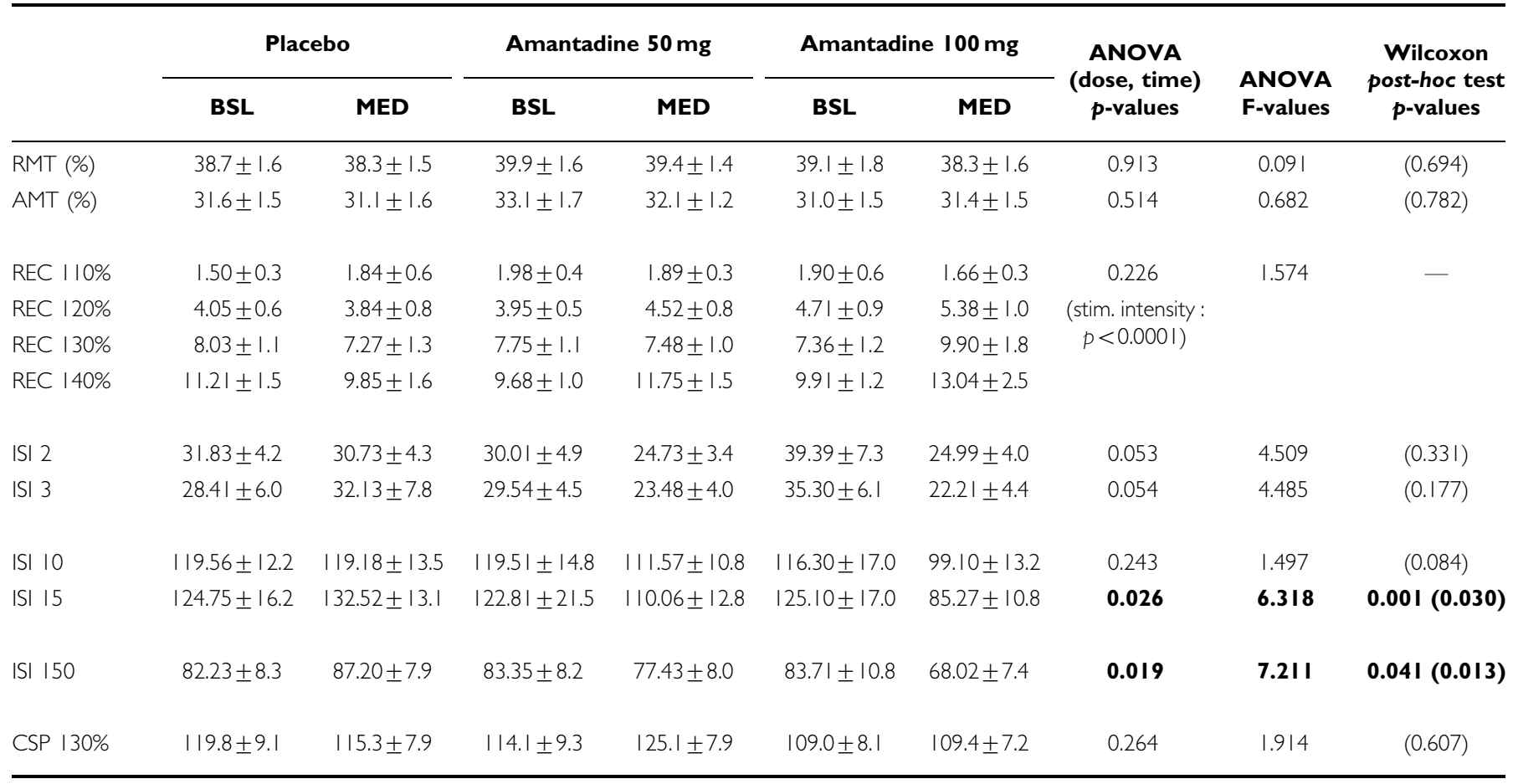

p-Values for the ANOVA for repeated measurements as well as for the adjusted post-hoc comparisons ( $100 \mathrm{mg}$ amantadine vs baseline and placebo (parenthesized)) are presented. Level of significance is set to $p<0.05$ for the ANOVA and for the Wilcoxon signed-rank test after Bonferroni-Holm adjustment.

Bold indicates significant results.

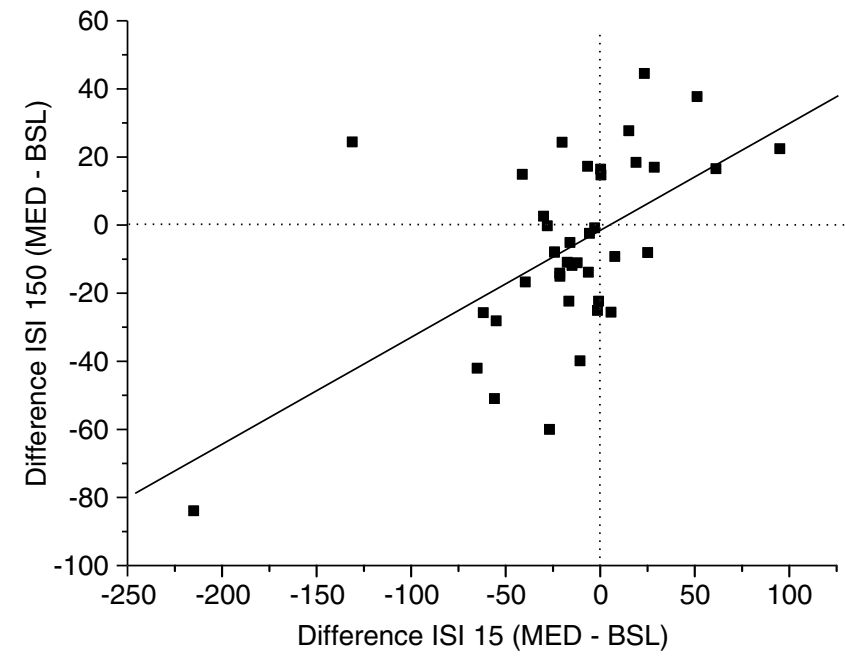

Figure 2 Correlation of TMS-evoked MEP response change at an ISI of I $5 \mathrm{~ms}$ and of ISI $150 \mathrm{~ms}$. Two-sided Kendall-Tau-b correlation: $R=0.579$, $p<0.0001$. Values for ISI I5 and ISI I50 are expressed as change of conditioned MEP responses after $100 \mathrm{mg}$ of amantadine (\% response (MED) $-\%$ response (baseline)). Level of significance: $p<0.05$.

motor cortex excitability was found for both the ICF (as expressed by ISI $15 \mathrm{~ms}$ ) and the L-ICI (as expressed by ISI $150 \mathrm{~ms}$ ). The Kendall-Tau-b correlation was $R=-0.289$, $p=0.009$ for ICF and $R=-0.228, p=0.041$ for L-ICI (Figure 4).

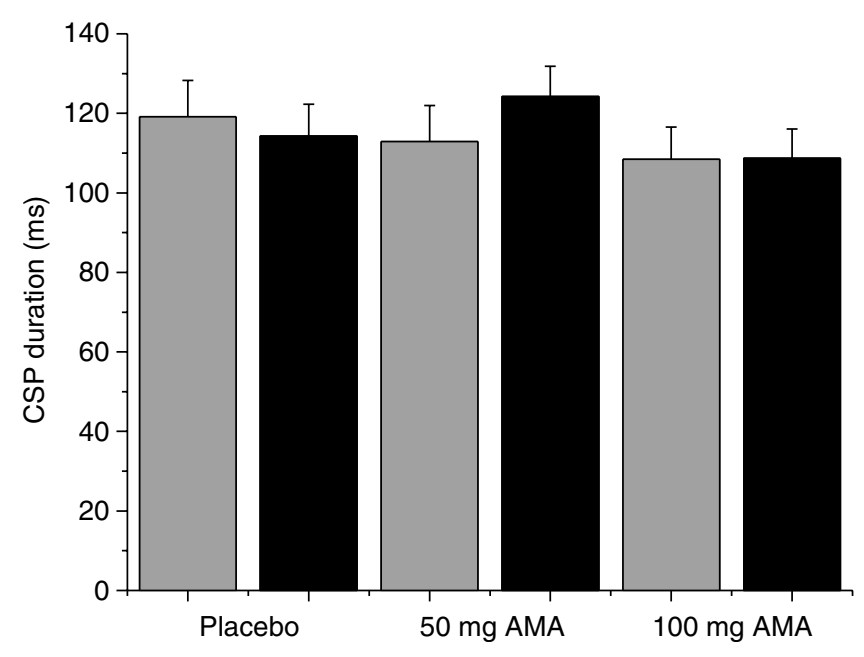

Figure 3 CSP duration (ms) at a stimulation intensity of $130 \%$ of AMT before (BSL) and after (MED) the administration of placebo and $50 \mathrm{mg}$ (50 mg AMA) and $100 \mathrm{mg}$ (100 mg AMA) amantadine. Data are given as mean $\pm \mathrm{SEM}$. No significant changes were observed after drug administration.

\section{Side Effects, Behavioral Parameters}

Amantadine was generally well tolerated. Following $50 \mathrm{mg}$ AMA, four subjects had mild CNS-related adverse effects starting about $30 \mathrm{~min}$ after drug intake and resolving within 

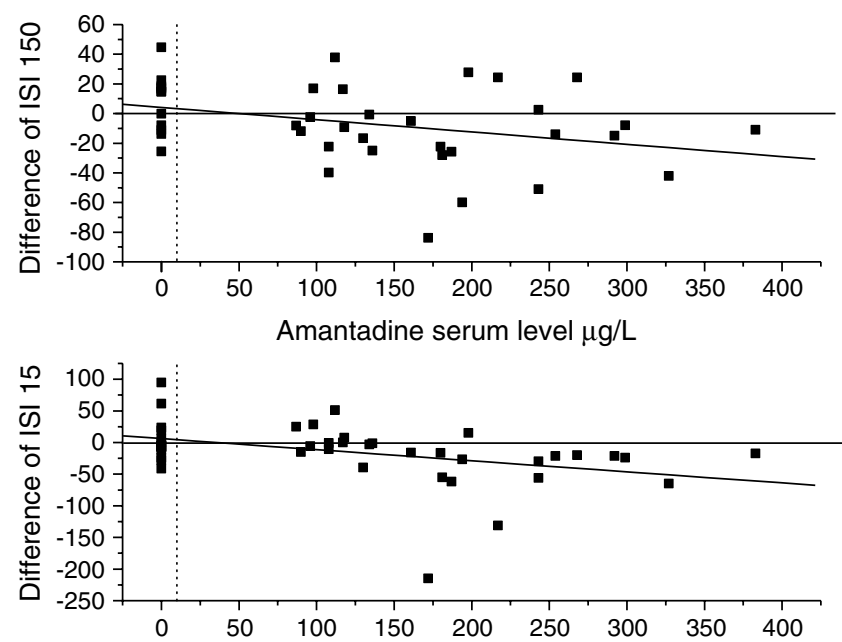

Figure 4 Correlation of amantadine serum concentration and change of average response to paired TMS at an ISI of I $50 \mathrm{~ms}$ (upper graph) and of ISI $15 \mathrm{~ms}$ (bottom graph). Two-sided Kendall-Tau-b correlation: $R=-0.289, p=0.009$ for $|S| \mid 5$ and $R=-0.228, p=0.04 \mid$ for $|S| \mid 50$. Serum concentrations are given in $\mu \mathrm{g} /$; Values for ISI 15 and ISI 150 are expressed as change of conditioned MEP responses after amantadine (\% response (MED)-\% response (baseline)). Level of significance: $p<0.05$.

$4 \mathrm{~h}$ (restlessness, headache, cognitive disturbance, visual disturbance). The intensity of these effects had no influence on the subjects' ability to complete the study. Interestingly, after a dose of $100 \mathrm{mg}$ AMA no subject reported adverse effects. Average side effect scores were 0 (placebo), $0.010 \pm 0.006$ ( $50 \mathrm{mg}$ AMA), and 0 (100 mg AMA). Thus, unblinding of the investigators and the subjects for the given dose seems to be unlikely. The results of the grooved pegboard test as a measure of motor dexterity and visualmotor coordination for the dominant and nondominant hand did not significantly differ after all three drug interventions as compared to baseline (dominant hand: placebo $+1 \mathrm{~s}, 50 \mathrm{mg}$ AMA $+2 \mathrm{~s}, 100 \mathrm{mg}$ AMA $-1 \mathrm{~s}$; nondominant hand: placebo $-1 \mathrm{~s}, 50 \mathrm{mg}$ AMA $+1 \mathrm{~s}$, $100 \mathrm{mg}$ AMA $-3 \mathrm{~s}$ difference from baseline (time to complete the test))

\section{DISCUSSION}

This is the first study to explore the effects of AMA on motor cortex excitability in healthy subjects. The main result of the present study is that a single oral dose of AMA significantly decreases ICF and increases L-ICI in the motor cortex. Furthermore, AMA showed a nonsignificant increase of short ICI. MEP recruitment curves, motor thresholds, the duration of the CSP, and peripheral neuronal excitability remained unchanged after treatment. Unexpectedly, a dissociation of L-ICI and CSP was found.

\section{Effect of AMA on Motor Thresholds and Recruitment Curves}

As a rule, motor thresholds are thought to reflect membrane excitability in pyramidal neurons, which is controlled by voltage-gated ion channels. Support for this view is provided by TMS studies of antiepileptic drugs with prominent sodium- and calcium-channel blocking but limited or no neurotransmitter interaction (Ziemann et al, 1996a). For example, carbamazepine and levetiracetam increase RMT and AMT and lead to a suppression of MEP recruitment curves (Reis et al, 2004; Ziemann et al, 1996a). Regardless of the involvement of sodium or calcium channel, the effects observed with TMS were comparable (Reis et al, 2004; Ziemann et al, 1996a).

The fact that neither the motor thresholds nor the test MEP amplitudes were altered by AMA suggests that an alteration of neuronal membrane excitability via ion channels is not of major relevance for its M1 excitability modulating effect. Furthermore, tiredness, attentional deficits, or motor slowing could be associated with an overall decrease of cortical excitability (Hess et al, 1987) and vice versa (Hadj Tahar et al, 2004). During this study, no sedating side effects were reported and the motor performance as evaluated by the grooved pegboard test did not change after drug intake. Therefore, as neither sedative effects nor threshold changes explored by TMS were present, it was assumed that AMA does not induce a general decrease of cortical excitability.

\section{Effect of AMA on Short ICI and Facilitation}

In the present study, AMA significantly decreased ICF and tended to increase ICI. These parameters are thought to reflect the activation of groups of excitatory and inhibitory cortical interneurons affecting the motor cortex and show different response to pharmacological interventions supporting the idea that these parameters are independent. Although ICI is in part regulated by GABA via postsynaptic $\mathrm{GABA}_{\mathrm{A}}$-receptor activation (Kujirai et al, 1993), ICF is probably the more complex phenomenon in which more than one neurotransmitter (glutamate, dopamine, GABA) may be involved (Nakamura et al, 1997; Ziemann et al, 1996a). The application of antiglutamatergic drugs like riluzole, memantine, or dextromethorphan predominantly leads to a suppression of ICF (Liepert et al, 1997; Schwenkreis et al, 1999, 2000). This suppression is-to a lesser extent - also present after application of GABAergic drugs such as vigabatrin, baclofen, or gabapentin (Ziemann et al, 1996a) or dopaminergic agents (Ziemann et al, 1997). AMA inhibits NMDA responses at clinically used concentrations (below $100 \mu \mathrm{M}$ ). The mechanism of action at the glutamatergic synapse is different from that of other NMDA antagonists characterized as 'open-channel blockers', as AMA mostly acts by stabilization of channel-closed states (Blanpied et al, 2005). However, regarding the effects on motor cortex excitability there seems to be no difference between AMA and other NMDA receptor antagonists as determined by comparable changes of both ICF and ICI. The NMDA receptor antagonists dextromethorphan, memantine, and riluzole predominantly decrease ICF and to a lesser extent increase ICI (Liepert et al, 1997; Schwenkreis et al, 1999; Ziemann et al, 1998). The effect of riluzole and memantine negatively correlated with its plasma levels in case of ICF changes (Schwenkreis et al, 1999, 2000). For memantine, a correlation with plasma levels was also shown for ICI (Schwenkreis et al, 1999). In concordance with these results, our study showed a significant correlation between 
changes of ICF and AMA serum levels, supporting the assumed dose-dependent antiglutamatergic effects of AMA.

Aside from the TMS studies, further evidence for an NMDA-antagonistic mechanism of action of AMA is provided by behavioral studies of motor learning in healthy volunteers: Hadj Tahar et al (2004) investigated the effect of $200 \mathrm{mg}$ AMA on motor learning, and found a worsening of motor performance in the AMA-treated group compared to placebo. Comparable results have been shown for the effect of the NMDA-antagonist memantine on motor learning, especially after continuous application for 8 days (Schwenkreis et al, 2005). It is assumed that use-dependent plasticity during motor learning is analogous to synaptic long-term potentiation (LTP) including the crucial role of NMDA receptors for LTP induction. (Butefisch et al, 2002; Ziemann et al, 1998). Hence, LTP can be prevented by the concurrent administration of NMDA-antagonists such as dextromethorphan (Ziemann et al, 1998) or memantine (Schwenkreis et al, 2005), and the results of Hadj Tahar et al (2004) support the view, that AMA predominantly acts via NMDA receptor antagonism, possibly at the level of the striatum.

In addition, the effect of AMA on the DA system in in vitro and in vivo experiments is an increased striatal DA activity (Shannon et al, 1987; Deep et al, 1999; Moresco et al, 2002).

However, this mechanism of action seems to be dosedependent and only relevant in higher doses exceeding the dose given in our study and those achieved in clinical use (Jackisch et al, 1992; Lupp et al, 1992). As it has been shown that antidopaminergic drugs like haloperidol lead to an increase of ICF and a decrease of ICI and dopaminergic substances produce converse effects (Ziemann et al, 1997), a dopaminergic effect of AMA could theoretically explain the detected decrease of ICF. However, with the low therapeutic dose applied in our study the effect of AMA on M1 excitability is likely unrelated to a relevant enhancement of dopaminergic transmission. Furthermore, with comparably low AMA plasma levels in the study by Hadj Tahar et al (2004), dopaminergic effects were judged as improbable, supported by the fact, that dopaminergic drugs have been shown to improve motor learning (Butefisch et al, 2002; Ziemann et al, 2002) and a worsening of motor learning was shown for AMA.

\section{Effects of AMA on Late ICI and CSP}

Single doses of AMA led to a significant increase of L-ICI without influencing the CSP.

Nakamura et al (1997) found a positive correlation between the extent of L-ICI and the duration of the CSP in normal subjects, suggesting a close relationship between the two phenomena. Because the CSP follows a similar time course as the late $\mathrm{GABA}_{\mathrm{B}}$-receptor-induced component of the inhibitory postsynaptic potential, it has been suggested that the later part of the CSP reflects activation of postsynaptic $\mathrm{GABA}_{\mathrm{B}}$-receptors (Hallett, 1995), presumably on intracortical interneurons (Tergau et al, 1999). This is supported by a study showing a CSP prolongation after application of the selective $\mathrm{GABA}_{\mathrm{B}}$-receptor agonist baclofen (Siebner et al, 1998). Other less specific GABA-ergic drugs, for example, lorazepam and valproate, also produce a lengthening of the CSP (Ziemann et al, 1996a,b).

As recently documented by epidural monitoring of TMSevoked corticospinal volleys, the L-ICI at ISIs of 70-150 ms, expressed as the strong suppression of a test MEP by an equal suprathreshold conditioning stimulus, also depends at least in part on $\mathrm{GABA}_{\mathrm{B}}$-ergic mechanisms at the cortical level as documented by I3 wave inhibition (Nakamura et al, 1997) whereas spinal inhibition was ruled out at this time interval owing to normal $\mathrm{H}$ reflex testing (Fuhr et al, 1991). A recent publication showing that the selective $G_{A B A}$ receptor agonist baclofen significantly increases L-ICI supports this point of view (McDonnell et al, 2006). However, in this study a positive correlation between the extent of L-ICI and the duration of the CSP was not found after intake of baclofen (McDonnell et al, 2006). In their study the L-ICI was significantly increased by the drug, whereas the CSP remained unchanged. The result suggested different underlying intracortical processes as L-ICI possibly reflects the magnitude of inhibition and CSP reflects the duration of inhibition.

AMA did not affect the CSP in the present study. This was expected, in concordance with previous studies with antiglutamatergic drugs such as riluzole and memantine, which did not evoke any change of this inhibitory phenomenon (Liepert et al, 1997; Schwenkreis et al, 1999).

Unexpectedly single doses of AMA led to a significant and dose-dependent increase of L-ICI. As L-ICI has not been investigated in the previous studies on NMDA antagonists (Liepert et al, 1997; Schwenkreis et al, 1999, 2000), comparability of the result is limited. There, however, are several possible explanations for this phenomenon:

Taking the time course of both TMS parameters into account (CSP length approximately $115 \mathrm{~ms}$ and L-ICI measured at ISI $150 \mathrm{~ms}$ ), the most reasonable explanation for significant changes of L-ICI seems to be a polysynaptic pathway, possibly involving in part the NMDA-receptormediated late EPSPs occurring at a similar time point, which could be inhibited by AMA and result in an increased inhibitory drive (Nakanishi et al, 1990). The significant correlation of ICF and L-ICI changes after AMA also suggests a strong relationship between both parameters.

Furthermore, the polysynaptic pathway may secondarily alter GABAergic interneurons. Supported by the results of McDonnell et al (2006), a separate influence of AMA on the magnitude of inhibition at a single time point (150 ms after a first stimulus) without affecting the duration of inhibition is assumed.

As it has been shown that dopaminergic drugs lead to inconsistent bidirectional changes of the L-ICI in PD patients (Lefaucheur, 2005; Berardelli et al, 1996; Pierantozzi et al, 2001; Chen et al, 2001) and AMA may have dopaminergic effects at high doses, a direct action via the dopaminergic system cannot be completely ruled out, but seems to be unlikely due to low serum levels measured. However, in this case a prolongation of the CSP would have been expected, because other dopaminergic drugs significantly increase the CSP duration in patients and healthy volunteers (Lefaucheur, 2005; Ziemann et al, 1996c).

In summary, the results described above provide evidence for NMDA-mediated excitability changes, which may also result in polysynaptic changes of GABA-ergic transmission. 


\section{CONCLUSION}

Our data suggest that single doses of 50-100 mg amantadine modulate motor cortex excitability predominantly by a reduction of NMDA receptor-mediated excitation and a complex polysynaptic interaction, possibly involving GABA-ergic transmission.

To clarify the observed dissociation of CSP and L-ICI and to investigate whether L-ICI may be helpful to gain insight into glutamatergic pathways, TMS studies in healthy subjects comparing NMDA antagonists and GABA agonists may be promising. Furthermore, it would be an interesting parameter for use in studies of use-dependent plasticity during motor tasks.

\section{ACKNOWLEDGEMENTS}

We thank B Fritsch and A Haag for their helpful comments and suggestions. This work was partially supported by the ULRAN-Foundation Professorship for Neurology/Epilepto$\operatorname{logy}(\mathrm{FR})$.

\section{REFERENCES}

Aoki FY, Sitar DS (1985). Amantadine kinetics in healthy elderly men: implications for influenza prevention. Clin Pharmacol Ther 37: 137-144.

Aoki FY, Sitar DS, Ogilvie RI (1979). Amantadine kinetics in healthy young subjects after long-term dosing. Clin Pharmacol Ther 26: 729-736.

Arndt T, Guessregen B, Hohl A, Reis J (2005). Determination of serum amantadine by liquid chromatography-tandem mass spectrometry. Clin Chim Acta 359: 125-131.

Berardelli A, Rona S, Inghilleri M, Manfredi M (1996). Cortical inhibition in Parkinson's disease. A study with paired magnetic stimulation. Brain 119(Part 1): 71-77.

Blanpied TA, Clarke RJ, Johnson JW (2005). Amantadine inhibits NMDA receptors by accelerating channel closure during channel block. J Neurosci 25: 3312-3322.

Brasil-Neto JP, McShane LM, Fuhr P, Hallett M, Cohen LG (1992). Topographic mapping of the human motor cortex with magnetic stimulation: factors affecting accuracy and reproducibility. Electroencephalogr Clin Neurophysiol 85: 9-16.

Butefisch CM, Davis BC, Sawaki L, Waldvogel D, Classen J, Kopylev L et al (2002). Modulation of use-dependent plasticity by d-amphetamine. Ann Neurol 51: 59-68.

Chen R, Kumar S, Garg RR, Lang AE (2001). Impairment of motor cortex activation and deactivation in Parkinson's disease. Clin Neurophysiol 112: 600-607.

Deep P, Dagher A, Sadikot A, Gjedde A, Cumming P (1999). Stimulation of dopa decarboxylase activity in striatum of healthy human brain secondary to NMDA receptor antagonism with a low dose of amantadine. Synapse 34: 313-318.

DiLazzaro V, Restuccia D, Oliviero A, Profice P, Ferrara L, Insola A et al (1998). Magnetic transcranial stimulation at intensities below active motor threshold activates intracortical inhibitory circuits. Exp Brain Res 119: 265-268.

Farnebo LO, Fuxe K, Goldstein M, Hamberger B, Ungerstedt U (1971). Dopamine and noradrenaline releasing action of amantadine in the central and peripheral nervous system: a possible mode of action in Parkinson's disease. Eur J Pharmacol 16: $27-38$.

Fuhr P, Agostino R, Hallett M (1991). Spinal motor neuron excitability during the silent period after cortical stimulation. Electroencephalogr Clin Neurophysiol 81: 257-262.
Hadj Tahar A, Blanchet PJ, Doyon J (2004). Motor-learning impairment by amantadine in healthy volunteers. Neuropsychopharmacology 29: 187-194.

Hallett M (1995). Transcranial magnetic stimulation. Negative effects. Adv Neurol 67: 107-113.

Hamer HM, Reis J, Mueller HH, Knake S, Overhof M, Oertel WH et al (2005). Motor cortex excitability in focal epilepsies not including the primary motor area-a TMS study. Brain 128: 811-818.

Heikkila RE, Cohen G (1972). Evaluation of amantadine as a releasing agent or uptake blocker for $\mathrm{H} 3$-dopamine in rat brain slices. Eur J Pharmacol 20: 156-160.

Hess CW, Mills KR, Murray NM, Schriefer TN (1987). Excitability of the human motor cortex is enhanced during REM sleep. Neurosci Lett 82: 47-52.

Jackisch R, Link T, Neufang B, Koch R (1992). Studies on the mechanism of action of the antiparkinsonian drugs memantine and amantadine: no evidence for direct dopaminomimetic or antimuscarinic properties. Arch Int Pharmacodyn Ther 320: $21-42$.

Kornhuber J, Bormann J, Hubers M, Rusche K, Riederer P (1991). Effects of the 1-amino-adamantanes at the MK-801-binding site of the NMDA-receptor-gated ion channel: a human postmortem brain study. Eur J Pharmacol 206: 297-300.

Kornhuber J, Quack G, Danysz W, Jellinger K, Danielczyk W, Gsell W et al (1995). Therapeutic brain concentration of the NMDA receptor antagonist amantadine. Neuropharmacology 34: 713-721.

Kornhuber J, Schoppmeyer K, Riederer P (1993a). Affinity of 1-aminoadamantanes for the sigma binding site in post-mortem human frontal cortex. Neurosci Lett 163: 129-131.

Kornhuber J, Weller M, Riederer P (1993b). Glutamate receptor antagonists for neuroleptic malignant syndrome and akinetic hyperthermic parkinsonian crisis. J Neural Transm Park Dis Dement Sect 6: 63-72.

Kujirai T, Caramia MD, Rothwell JC, Day BL, Thompson PD, Ferbert A et al (1993). Corticocortical inhibition in human motor cortex. J Physiol 471: 501-519.

Lefaucheur JP (2005). Motor cortex dysfunction revealed by cortical excitability studies in Parkinson's disease: influence of antiparkinsonian treatment and cortical stimulation. Clin Neurophysiol 116: 244-253.

Liepert J, Schwenkreis P, Tegenthoff M, Malin JP (1997). The glutamate antagonist riluzole suppresses intracortical facilitation. J Neural Transm 104: 1207-1214.

Lin TI, Heider H, Schroeder C (1997). Different modes of inhibition by adamantane amine derivatives and natural polyamines of the functionally reconstituted influenza virus M2 proton channel protein. J Gen Virol 78(Part 4): 767-774.

Lupp A, Lucking CH, Koch R, Jackisch R, Feuerstein TJ (1992). Inhibitory effects of the antiparkinsonian drugs memantine and amantadine on $N$-methyl-D-aspartate-evoked acetylcholine release in the rabbit caudate nucleus in vitro. J Pharmacol Exp Ther 263: 717-724.

Matsubayashi H, Swanson KL, Albuquerque EX (1997). Amantadine inhibits nicotinic acetylcholine receptor function in hippocampal neurons. J Pharmacol Exp Ther 281: 834-844.

McDonnell MN, Orekhov Y, Ziemann U (2006). The role of $\mathrm{GABA}(\mathrm{B})$ receptors in intracortical inhibition in the human motor cortex. Exp Brain Res [E-pub ahead of print].

Mizoguchi K, Yokoo H, Yoshida M, Tanaka T, Tanaka M (1994). Amantadine increases the extracellular dopamine levels in the striatum by re-uptake inhibition and by $N$-methyl-D-aspartate antagonism. Brain Res 662: 255-258.

Moresco RM, Volonte MA, Messa C, Gobbo C, Galli L, Carpinelli A et al (2002). New perspectives on neurochemical effects of amantadine in the brain of parkinsonian patients: a PET-[(11)C] raclopride study. J Neural Transm 109: 1265-1274. 
Nakamura H, Kitagawa H, Kawaguchi Y, Tsuji H (1997). Intracortical facilitation and inhibition after transcranial magnetic stimulation in conscious humans. J Physiol 498(Part 3): 817-823.

Nakanishi H, Ukai K, Nakagawa T, Watanabe S, Kamata O, Yamamoto K (1990). Enhancement of NMDA receptor-mediated synaptic potential evoked in rat medial-amygdala neuron following olfactory bulbectomy. Brain Res 532: 69-75.

Northover BJ (1994). Effect of pre-treating rat atria with potassium channel blocking drugs on the electrical and mechanical responses to phenylephrine. Biochem Pharmacol 47: 2163-2169.

Oldfield RC (1971). The assessment and analysis of handedness: the Edinburgh inventory. Neuropsychologia 9: 97-113.

Parkes D (1974). Amantadine. Adv Drug Res 8: 11-81.

Parsons CG, Panchenko VA, Pinchenko VO, Tsyndrenko AY, Krishtal OA (1996). Comparative patch-clamp studies with freshly dissociated rat hippocampal and striatal neurons on the NMDA receptor antagonistic effects of amantadine and memantine. Eur J Neurosci 8: 446-454.

Pascual-Leone A, Cohen LG, Brasil-Neto JP, Hallett M (1994). Non-invasive differentiation of motor cortical representation of hand muscles by mapping of optimal current directions. Electroencephalogr Clin Neurophysiol 93: 42-48.

Pierantozzi M, Palmieri MG, Marciani MG, Bernardi G, Giacomini P, Stanzione P (2001). Effect of apomorphine on cortical inhibition in Parkinson's disease patients: a transcranial magnetic stimulation study. Exp Brain Res 141: 52-62.

Priori A, Berardelli A, Inghilleri M, Accornero N, Manfredi M (1994). Motor cortical inhibition and the dopaminergic system. Pharmacological changes in the silent period after transcranial brain stimulation in normal subjects, patients with Parkinson's disease and drug-induced parkinsonism. Brain 117(Part 2): 317-323.

Reis J, Tergau F, Hamer HM, Muller HH, Knake S, Fritsch B et al (2002). Topiramate selectively decreases intracortical excitability in human motor cortex. Epilepsia 43: 1149-1156.

Reis J, Wentrup A, Hamer HM, Mueller HH, Knake S, Tergau F et al (2004). Levetiracetam influences human motor cortex excitability mainly by modulation of ion channel function - a TMS study. Epilepsy Res 62: 41-51.

Schwenkreis P, Liepert J, Witscher K, Fischer W, Weiller C, Malin JP et al (2000). Riluzole suppresses motor cortex facilitation in correlation to its plasma level. A study using transcranial magnetic stimulation. Exp Brain Res 135: 293-299.

Schwenkreis P, Witscher K, Janssen F, Addo A, Dertwinkel R, Zenz $\mathrm{M}$ et al (1999). Influence of the $N$-methyl-D-aspartate antagonist memantine on human motor cortex excitability. Neurosci Lett 270: $137-140$.

Schwenkreis P, Witscher K, Pleger B, Malin JP, Tegenthoff M (2005). The NMDA antagonist memantine affects training induced motor cortex plasticity - a study using transcranial magnetic stimulation. BMC Neurosci 12: 35.

Shannon KM, Goetz CG, Carroll VS, Tanner CM, Klawans HL (1987). Amantadine and motor fluctuations in chronic Parkinson's disease. Clin Neuropharmacol 10: 522-526.
Siebner HR, Dressnandt J, Auer C, Conrad B (1998). Continuous intrathecal baclofen infusions induced a marked increase of the transcranially evoked silent period in a patient with generalized dystonia. Muscle Nerve 21: 1209-1212.

Stoof JC, Booij J, Drukarch B, Wolters EC (1992). The antiparkinsonian drug amantadine inhibits the $N$-methyl-D-aspartic acid-evoked release of acetylcholine from rat neostriatum in a non-competitive way. Eur J Pharmacol 213: 439-443.

Takahashi T, Yamashita H, Zhang YX, Nakamura S (1996). Inhibitory effect of MK-801 on amantadine-induced dopamine release in the rat striatum. Brain Res Bull 41: 363-367.

Takeda M, Pekosz A, Shuck K, Pinto LH, Lamb RA (2002). Influenza a virus $\mathrm{M} 2$ ion channel activity is essential for efficient replication in tissue culture. J Virol 76: 1391-1399.

Tergau F, Wanschura V, Canelo M, Wischer S, Wassermann EM, Ziemann U et al (1999). Complete suppression of voluntary motor drive during the silent period after transcranial magnetic stimulation. Exp Brain Res 124: 447-454.

Togo Y, Hornick RB, Dawkins Jr AT (1968). Studies on induced influenza in man. I. Double-blind studies designed to assess prophylactic efficacy of amantadine hydrochloride against a2/ Rockville/1/65 strain. JAMA 203: 1089-1094.

Verhagen ML, Del Dotto P, van den MP, Fang J, Mouradian MM, Chase TN (1998). Amantadine as treatment for dyskinesias and motor fluctuations in Parkinson's disease. Neurology 50: 1323-1326.

Vernier VG, Harmon JB, Stump JM, Lynes TE, Marvel JP, Smith DH (1969). The toxicologic and pharmacologic properties of amantadine hydrochloride. Toxicol Appl Pharmacol 15: 642-665.

Werhahn KJ, Kunesch E, Noachtar S, Benecke R, Classen J (1999). Differential effects on motorcortical inhibition induced by blockade of GABA uptake in humans. J Physiol 517(Part 2): 591-597.

Ziemann U, Bruns D, Paulus W (1996c). Enhancement of human motor cortex inhibition by the dopamine receptor agonist pergolide: evidence from transcranial magnetic stimulation. Neurosci Lett 208: 187-190.

Ziemann U, Chen R, Cohen LG, Hallett M (1998). Dextromethorphan decreases the excitability of the human motor cortex. Neurology 51: 1320-1324.

Ziemann U, Lonnecker S, Steinhoff BJ, Paulus W (1996a). Effects of antiepileptic drugs on motor cortex excitability in humans: a transcranial magnetic stimulation study. Ann Neurol 40: 367-378.

Ziemann U, Lonnecker S, Steinhoff BJ, Paulus W (1996b). The effect of lorazepam on the motor cortical excitability in man. Exp Brain Res 109: 127-135.

Ziemann U, Tam A, Butefisch C, Cohen LG (2002). Dual modulating effects of amphetamine on neuronal excitability and stimulation-induced plasticity in human motor cortex. Clin Neurophysiol 113: 1308-1315.

Ziemann U, Tergau F, Bruns D, Baudewig J, Paulus W (1997). Changes in human motor cortex excitability induced by dopaminergic and anti-dopaminergic drugs. Electroencephalogr Clin Neurophysiol 105: 430-437. 